\title{
Utilización de hueso craneal particulado de calota para prevención de defectos óseos en la cirugía de las craneosinostosis
}

I. Solís; L. Miragall; V. Bordes*; G. Pérez-Herrezuelo; J.G. Darder*; J.V. Pascual; R. Guijarro; R. Villar; M.E. Iglesias; M. Puche y M. Marqués

Servicio de Cirugía Maxilofacial. Hospital Clínico Universitario de Valencia. *Servicio de Neurocirugía Hospital Clínico de Valencia. Valencia.

Resumen

Se considera que hasta un $20 \%$ de intervenidos de craneosinostosis requieren cirugías secundarias. Son varias las técnicas que se han venido utilizando dentro de la cirugía craneofacial para la reconstrucción de grandes defectos óseos en pacientes pediátricos a lo largo de los años. Presentamos una nueva técnica de obtención de injerto de hueso para el recubrimiento de defectos óseos craneales, en la que se utiliza hueso particulado, obtenido de la calota del paciente mediante un berbiquí y unificado con un adhesivo de fibrina. Está técnica es sencilla y provoca poca morbilidad en el paciente. Además, permite obtener una importante cantidad de hueso.

Desde el año 2007 utilizamos el hueso particulado autólogo obtenido de la calota del paciente para la corrección de defectos óseos grandes o pequeños que se nos presentaban en la cirugía craneofacial practicada sobre todo en pacientes pediátricos.

Aunque los materiales aloplásticos y sustitutos de hueso han sido utilizados para la reconstrucción de cráneos, el hueso autógeno es la mejor opción. A diferencia de los materiales sintéticos, los injertos autógenos tienen una más rápida osteointegración ya que son osteogénicos, osteoinductivos y osteoconductivos, siendo además el injerto de la misma naturaleza que el hueso donante. La morbilidad producida al paciente por la obtención del hueso de la calota con esta técnica es mínima, en comparación con otras zonas donantes como costilla o cadera.

La utilización del hueso particulado autólogo durante la cirugía de las craneosinostosis reduce la necesidad de segundas intervenciones por defectos de osificación secundarios. Por otro lado, su obtención es fácil y la cantidad de hueso extraído es suficiente para los pacientes pediátricos.

PALABRAS CLAVE: Cirugía craneofacial. Injerto particu-

Recibido: 20-09-09. Aceptado: 7-10-09 lado. Defectos óseos. Tratamiento. Tissucol.

Prevention of osseous defaults in the craneosinostosis surgery using calvarian cranial particulate bone

Summary

It is considered that up to $20 \%$ of the craniosinostosis patients require secondary surgeries. Different techniques have been used in craneofacial surgery for the reconstruction of great osseous defects in pediatric patients for many years. This paper is about a new technique to obtain osseous graft for covering osseous cranial defects, using particulate bone, harvested from the patient calvarian using a hand-driven brace and covered with a fibrin adhesive. This is a very simple technique, which provides a great amount of bone from the patient himself, therefore producing a small morbidity.

Since 2007 the authors have been using autologous particulate bone harvested from de patient calvarian for the reconstruction of different size osseous defects found in craneofacial surgery, especially in pediatrics patients.

Although alloplastic materials and bone substitutes have been used for cranial reconstruction, the best option is the autogenous bone. In contrast to synthetic materials autologous grafts have a faster osteointegration, due to their osteogenic, osteoinductive and osteconductive properties. Harvesting the bone from the calvarian patient produces a minimal morbidity compared to the extraction of grafts from other donor sites such as rips or hip.

The use of autologous particulate bone in craniosinostosis surgery reduces the risk of second interventions due to secondary ossifications defects. On the other hand, the harvest is easy and the supply of bone it is enough in pediatric patients.

KEY WORDS: Craneofacial surgery. Particulate bone graft. Treatement. Tissucol. 


\section{Introducción}

Uno de los problemas más comunes en la cirugía craneofacial es la escasez de hueso para subsanar los defectos producidos por la movilización de fragmentos óseos en la bóveda craneal.

La opción reconstructiva clásica ha sido siempre la obtención de splits de calota del propio paciente para perfeccionar esos defectos. Sin embargo, en el caso de los niños pequeños esta técnica sólo puede utilizarse cuando alcanzan la suficiente madurez e incluso en esos casos, la intervención es complicada y peligrosa si se hace externamente desde la bóveda craneal, por ello se recurre a trabajar en un torno de banco, pero dicho procedimiento es igualmente complejo.

También en los niños de muy corta edad, la obtención de hueso de la cresta iliaca o de costilla se descarta en una primera cirugía y sólo se recurre a estos bancos óseos en caso de intervenciones secundarias reparadoras, cuando el paciente es más mayor. Sin embargo, aunque estas técnicas se utilizan, no son recomendadas debido a varios problemas: la rápida reabsorción en el caso de la costilla; la escasa cantidad de hueso donante en el de la cresta iliaca y la gran morbilidad que se produce en ambas situaciones.

Como puede observarse, según las técnicas de obtención de hueso hasta ahora existentes, nos encontrábamos con varios inconvenientes: en primer lugar, las escasas zonas donantes y, en segundo lugar, la incompatibilidad de las técnicas en pacientes de corta edad.

Por ello, trabajamos hasta encontrar una opción alternativa: el hueso particulado autólogo de la calota obtenido mediante un berbiquí. Así, se resuelven varios de los problemas ya planteados. La técnica, puede realizarse en pacientes de edades tempranas; la obtención de hueso tiene lugar en la primera operación, por tanto no habría que esperar a una segunda intervención; no se produce una gran morbilidad y se dispone de una cantidad aceptable de hueso que se puede adaptar a los diferentes defectos.

\section{Material y métodos}

Desde el año 2007 éste es un procedimiento habitual en todas nuestras cirugías de craneosinostosis, tanto las que incluyen necesidad de avance y remodelado de la barra frontoorbitaria (plagiocefalia, trigonocefalia, braquicefalia), las que corresponden a desmontajes holocraneales (escafocefalia, craneosinostosis múltiples), o aquéllas en las que se lleva a cabo remodelados y expansión de fosas posteriores.

En los casos de avances y remodelación frontoorbitaria la técnica que venimos presentando, la escogemos para evitar la depresión de la fosa temporal.

En el resto de la bóveda craneal, la utilizamos para dar

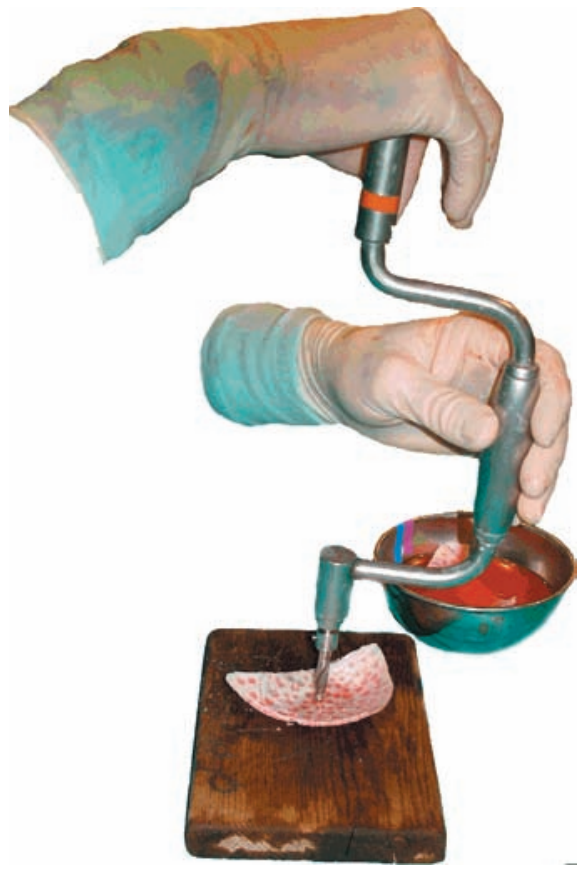

Figura 1. Observamos el berbiquí aplicado sobre el endocortex del diploe.

continuidad y evitar los espacios muertos entre los fragmentos óseos consiguiendo así prevenir escalones inestéticos.

Como ya se ha comentado anteriormente, para obtener hueso particulado, utilizamos un berbiquí y el tipo de broca escogida es la específica para craneotomía D'Errico (Codman). Con ello, se pretende no perforar completamente el espesor total de la calota sino realizar una impronta sobre el diploe de grosor parcial (es decir sólo cortical interna o cortical externa).

En la intervención de la craneosinostosis, se utilizan los fragmentos de hueso en el momento de la cirugía de banco, centrándonos en la cortical interna como zona clave de obtención. Se inicia la perforación realizando una compresión axial sobre la calota hasta que el borde de ataque de la broca ha hecho impronta, momento en el cual retiramos el berbiquí, mientras lo seguimos haciendo rotar. De este modo, adquirimos pequeñas escamas de calota. (Fig. 1)

Si bien este procedimiento lo podemos realizar también sobre la cortical externa de los huesos de la calota, preferimos siempre que sea posible, utilizar la cortical interna sobre el banco. Al hacerlo así se consiguen tres ventajas: en primer lugar, se obtiene una mayor cantidad de injerto ya que la disposición de la broca con la concavidad del endocortex es más amplia. En segundo lugar, se aumenta la plasticidad de los fragmentos para la reconstrucción, porque al contar la fresa con un mayor punto de apoyo, es más fácil la obtención. Y en tercer lugar, el procedimiento es mucho más seguro que si aplicáramos el endocortex directamente sobre la cabeza del paciente. 


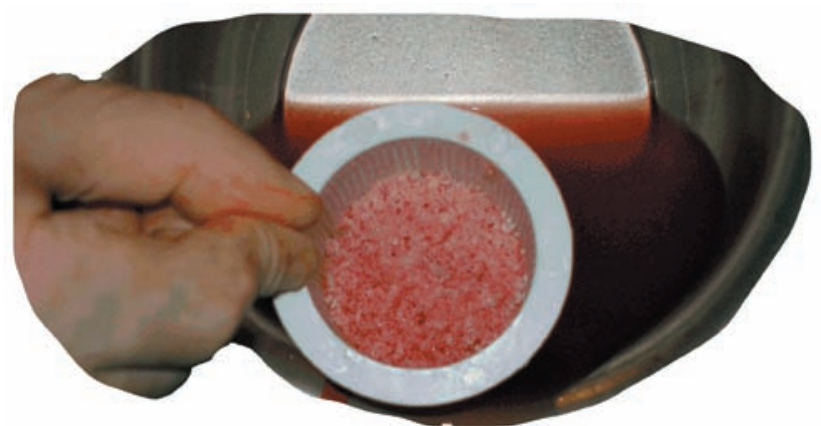

Figura 2. Filtrado del hueso particulado sobre un filtro urológico

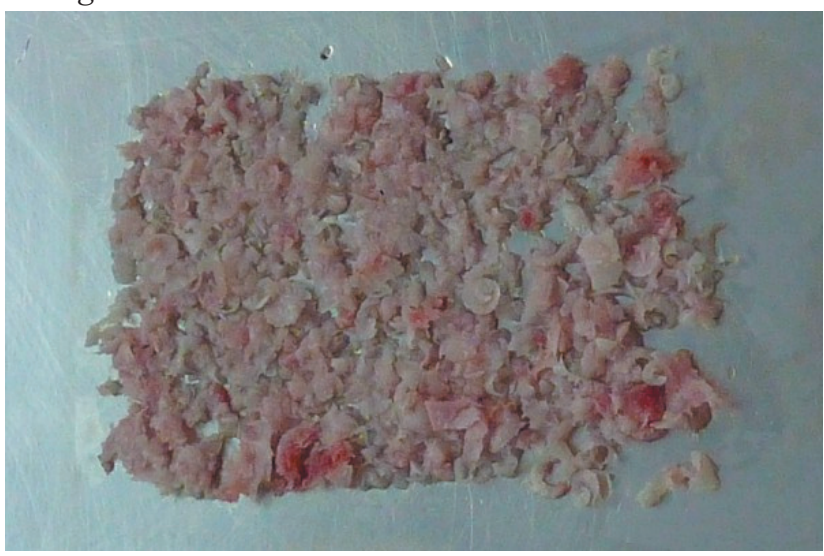

Figura 3. Plancha de hueso particulado previa a la aplicación del Tissucol.

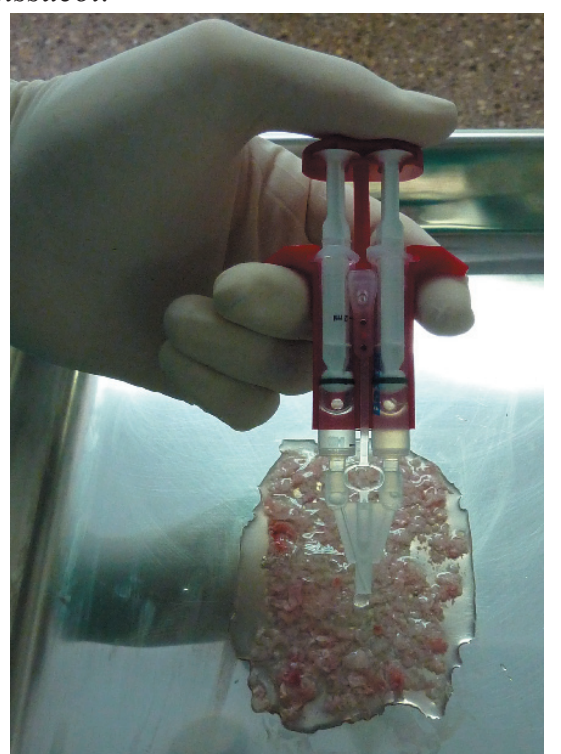

Figura 4. Aplicación de Tissucol sobre el hueso particulado.

Para la unificación del hueso y su posterior aplicación, utilizamos un adhesivo de fibrina (Tissucol ${ }^{\circledR}$, Beriplast ${ }^{\circledR}$ ...). Se filtra el hueso y, posteriormente, se extiende todo lo que sea posible sobre una superficie plana, siempre y
Neurocirugía 2010; 21: 118-124

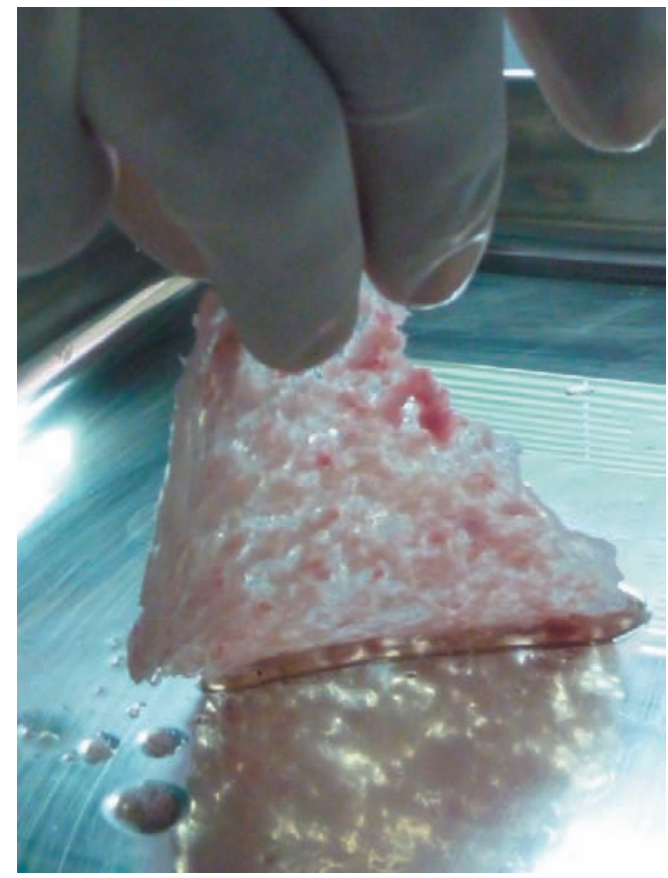

Figura 5. Aspecto de la consistencia del injerto una vez que el Tissucol empieza a fraguar.

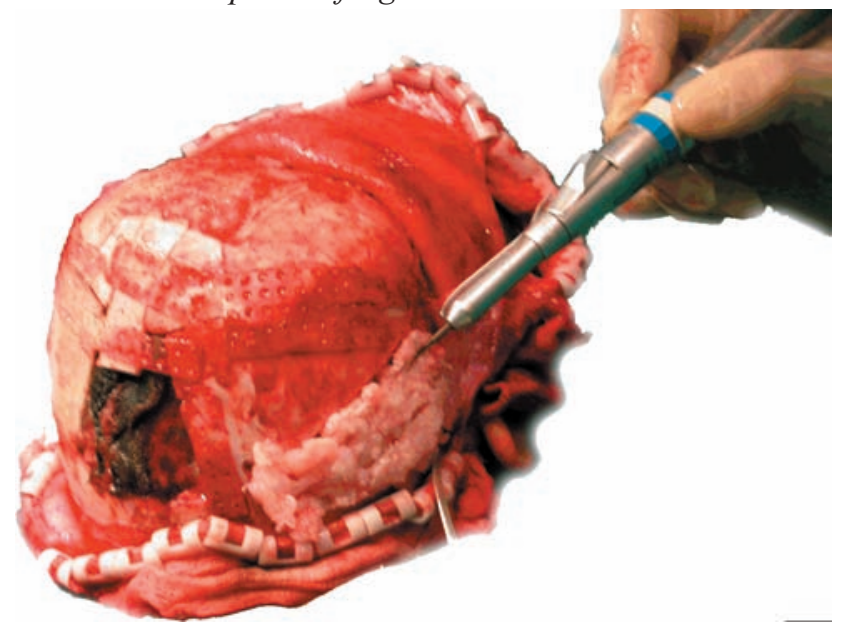

Figura 6. Paciente con defecto en fosa temporal una vez realizado avance fronto-orbitario. Vista final una vez aplicado el hueso sobre el defecto óseo.

cuando los fragmentos óseos estén en contacto. Se forma así una "fina plancha" ósea. Sobre esta superficie aplicamos el adhesivo de fibrina directamente, consiguiendo una película ósea de consistencia suficiente para poder ser aplicada sobre las zonas que deseemos. (Figs. 2- 4)

Esta "plancha" la podemos recortar para darle forma consiguiendo una correcta adaptación a los defectos óseos que teníamos. Es importante realizar este procedimiento de una forma relativamente rápida ya que el tiempo de fraguado de la fibrina no es mucho; disponiendo de escasos 7 minutos para el manipulado del injerto ${ }^{6,7}$. (Figs. 5, 6.) 


\section{Discusión}

Es bien conocido por todos el problema de la osificación de los huesos craneales después de la cirugía de las craneosinostosis. Aunque comúnmente consideramos que en niños pequeños menores de 18 meses la osificación craneal debe ser completa, después de nuestras cirugías, la experiencia nos demuestra repetidamente que esto no siempre es as ${ }^{19}$, y diversos estudios parecen indicar que cuando las cirugías se realizan por encima de los 9 meses de edad la posibilidad de reosificación incompleta alcanza el 50\%.

Algunos autores proponen realizar la cirugía de la craneosinostosis en edades muy tempranas, especialmente aquéllos que apoyan los avances frontoorbitarios, con los que se reducen considerablemente los defectos de osificación. No obstante, esto no siempre es posible debido a diagnósticos tardíos, patologías concomitantes que retrasan la intervención etc.

Por otro lado, la cirugía muy temprana conlleva alto riesgo anestésico ( 4,3 por 1000$)$, que se disminuye $(0,5$ por $1000)$ cuando se realiza cerca del año de edad ${ }^{9,16}$.

Además, nosotros creemos firmemente en una sólida fijación rígida en la cirugía de las craneosinostosis para mantener el contorno y un resultado predecible estético en el tiempo, tanto en los avances frontoorbitarios como en las expansiones craneales. El espesor de los huesos craneales es óptimo para esta fijación cuando el paciente se acerca al año de vida. Por último consideramos, como otros autores, que el riesgo de recidiva (en especial en los avances frontoorbitarios), es mucho menor cuando la cirugía se realiza cercana a la edad ya comentada ${ }^{17,18}$. Desde nuestro punto de vista, lo más recomendable es intervenir concretamente cuando el paciente cuenta con 9 meses (salvo que problemas neuroquirúrgicos indiquen la necesidad de cirugía más temprana), pues en esta edad se cuenta con un espesor del hueso craneal óptimo, existe menor riesgo anestésico y el potencial osteogénico de la duramadre no ha desaparecido.

Existen alternativas al hueso autólogo para la reconstrucción de defectos como son cementos óseos ${ }^{5,20}$, hueso desmineralizado ${ }^{1,13}$, polietileno poroso, hidroxiapatita ${ }^{15}$, metil metacrilato ${ }^{8,10}$ y proteínas adyuvantes osteoestimulantes ${ }^{14}$. Pero estas formas de hueso no autólogo conllevan el riesgo inherente de infección, extrusión, fragmentación, restricción del crecimiento, osificación patológica y reacciones inflamatorias ${ }^{2,8,10,11,20}$. Todas estas limitaciones nos han llevado a buscar un método ideal para la reconstrucción de grandes defectos craneales siendo, a día de hoy, el injerto autólogo la mejor alternativa ${ }^{3}$.

A diferencia de los materiales sintéticos, los injertos autógenos tienen una más rápida osteointegración ya que son osteogénicos, osteoinductivos, osteoconductivos y, por supuesto, el injerto es de la misma naturaleza que el hueso receptor.

En los pacientes pediátricos, proponemos la obtención desde la cortical interna durante la fase de cirugía de banco, lo que permite una extracción segura y al mismo tiempo disminuir el espesor de los fragmentos óseos y conseguir una mayor plasticidad de los mismos para la posterior craneoplastia estética, facilitándose además la revascularización de los fragmentos. Evitamos, por otro lado, la pérdida suplementaria de sangre que tendríamos si realizáramos la obtención de hueso del ectocortex sobre las regiones parietales tomadas como zona donante.

En nuestra experiencia, no hemos tenido problemas de infecciones postoperatorias o reabsorción de hueso relacionadas con este procedimiento. Los resultados estéticos son apreciables teniendo actualmente en nuestro ámbito una menor incidencia de osificaciones incompletas.

Somos conscientes de la limitación de estas afirmaciones ante la ausencia de TAC de control que corrobore la osificación óptima de este método, sin embargo, nos parece que es importante minimizar la radiación y las anestesias generales de nuestros pacientes ${ }^{12}$. Además, no hay que olvidar que en la práctica diaria los defectos de la bóveda craneal son fáciles de descubrir clínicamente, pues las fibrosis se deprimen con la presión y se puede apreciar la pulsación de la duramadre según el paciente coloque su cabeza.

\section{Conclusión}

El hueso particulado del ectocortex de calota es fácil de obtener con esta técnica; es especialmente moldeable para rellenar cualquier defecto y su obtención es segura. Reduce el riesgo de cirugías secundarias por defectos óseos residuales y elimina la morbilidad de la obtención de injerto óseo de otras zonas donantes como son la cresta iliaca o región costal.

Actualmente nosotros hemos extendido su uso también para adultos, cuando queremos solucionar grandes defectos óseos, siendo nuestra primera elección antes que los splits de calota.

\section{Bibliografía}

1. Acarturk, T.O., Hollinger, J.O.: Commercially available demineralized bone matrix compositions to regenerate calvarial critical-sized bone defects. Plastic \& Reconstructive Surgery. 2006; 118: 862-873.

2. Baker, S.B., Weinweig, J., Jirschner, R.E. et al.: Applications of a new carbonated calcium phosphate bone cement: Early experience in pediatric and adult craniofacial reconstruction Plastic \& Reconstructive Surgery. 2002; 190: 17891796.

3. Chao Mimi, T.M.D., Jiang, S.M.D., Smith, D.M.D. et al.: Demineralized Bone Matrix and Resorbable Mesh Bila- 
minate Cranioplasty: A Novel Method for Reconstruction of Large-Scale Defects in the Pediatric Calvaria Plastic \& Reconstructive Surgery. 2009; 123: 976-982.

4. Esparza, J., Hinojosa, J., García-Recuero, I., Romance, A., Pascual, B., Martínez de Aragón, A.: Surgical treatment of isolated and syndromic craniosynostosis. Results and complications in 283 consecutive cases. Neurocirugia 2008; 19: 509-529.

5. Greenberg, B.M., Schneider, S.J.: Alloplastic reconstruction of large cranio-orbital defects: A comparative evaluation. Ann Plast Surg 2005; 55: 43-51.

6. Greene, A.K., Mulliken, J.B., Proctor, M.R. et al.: Pediatric Cranioplasty Using Particulate Calvarial Bone Graft . Plastic \& Reconstructive Surgery. 2007; 122: 563-571.

7. Greene, A.K., Mulliken, J.B., Proctor, M.R. et al.: Primary Grafting with Autologous Cranial Particulate Bone Prevents Osseous Defects following Fronto-Orbital Advancement. Plastic \& Reconstructive Surgery. 2007: 120: 16031611.

8. Kaiser, A., Illi, O.E., Sailer, H.F., et al.: Cranioplasties for congenital and acquired skull defects in children: Comparison of new concepts with conventional methods Eur J Pediatr Surg. 1993; 3: 236-240.

9. Murat, I., Constant, I., Maudhuy, H.: Perioperative anaesthetic morbidity in children: Database of 24165 anaesthetics over 30-month period Paediatr. Anaesth 2004; 14: 158.

10. Pochon, J.P., Kloti, J.: Cranioplasty for acquired skull defect in children: A comparison between autologous material and methylmethacrylate Eur J Pediatr Surg 1991: 199-201.

11. Poetker, D.M., Pytynia, K.B., Meyer, G.A. et al.: Complication rate of transtemporal hydroxyapatite cement cranioplasties: A case series review of 76 cranioplasties Otol Neurol 2004; 25: 604-609.

12. Prevot, M., Renier, D., Marchac, D.: Lack of ossification after cranioplasty for craniosynostosis J Craniofac Surg 1993; 4: 247.

13. Salyer, K.E., Bardach, J., Squier, C.A. et al.: Cranioplasty in the growing canine skull using demineralized

\section{Comentario al trabajo Utilización de hueso craneal particulado de calota para prevención de defectos óseos en la cirugía de las craneosinostosis de I. Solís y cols.}

La cirugía craneofacial se caracteriza por el empleo de diferentes técnicas de remodelación de la calota, base craneal, órbitas y macizo facial. Todas ellas implican osteotomías múltiples con movilización y transposición de fragmentos óseos desvascularizados y un riesgo con- perforated bone. Plastic \& Reconstructive Surgery. 1995; 96: 770-779.

14. Smith, D.M., Cooper, G.M., Mooney, M.P. et al.: Bone morphogenetic protein 2 therapy for craniofacial surgery: A practical review. J Craniofac Surg 2008; 19: 12441259.

15. Staffa, G., Nataloni, A., Compagnone, C. et al.: Custom made cranioplasty prostheses in porous hydroxyl-apatite using three-dimensional design techniques: 7 years experience in 25 patients. Acta Neurochir 2007; 149: 161-170.

16. Tired, L., Nivoche, Y., Hatton, F. et al.: Complications related to anesthesia in infants and children. Br. J. Anaesth 1988; 61: 263.

17. Wall, S.A., Goldin, J.H., Hockley, A.D.: Fronto-orbital reparation in craniosynostosis. Br. J. Plast. Surg. 1994; 47: 180.

18. Whitaker, L.A., Bartlett, S.P., Schut, L. et al.: Craniosynostosis: an analysis of the timing, treatment, and complications in 164 consecutive patients. Plastic \& Reconstructive Surgery. 1987; 80: 195.

19. Wolfe, S.A., Rivas-Torres, M.T., Ozerdem, O.: Acquired facial bone deformities. Philadelphia: Elsevier, 2006. Pp. 564-565.

20. Zins, J.E., Moreira-Gonzalex, A., Papay, F.A.: Use of calcium-based bone cements in the repair of large, fullthickness cranial defects: A caution Plastic \& Reconstructive Surgery. 2007; 120: 1332-1342.

Solís, I.; Miragall, L.; Bordes, V.; Pérez-Herrezuelo, G.; Darder, J.V.; Pascual, J.V.; Guijarro, R.; Villar, R.; Iglesias, M.E.; Puche, M.; Marqués, M.: Utilización de hueso craneal particulado de calota para prevención de defectos óseos en la cirugía de las craneosinostosis. Neurocirugía 2010; 21: 118-124.

Correspondencia: Unidad de deformidades craneofaciales. Hospital Clinico De Valencia. Avda Blasco Ibáñez, 17. 46010 Valencia. España.

Email: solisignacio@gmail.com secuente de reabsorción o necrosis de los mismos y, por tanto, craneolacunias postoperatorias. Con frecuencia éstos pacientes necesitan de más de una intervención por la evolución de la enfermedad o tras un resultado insuficiente desde el punto de vista estético. La aparición de defec- 
tos craneales persistentes tras las mismas es una de las complicaciones más frecuentes en cirugía craneofacial y supone en ocasiones un formidable reto quirúrgico. Existen numerosos factores que pueden motivar la aparición de los defectos: el fracaso de la osteosíntesis (pseudoartrosis), la osteonecrosis por infección o factores inflamatorios locales, el déficit de osificación secundaria relacionada con cicatrices durales o la pérdida de periostio por técnica inadecuada, las cirugías de repetición, la edad elevada con la inherente disminución de la capacidad osteogénica, la hipertensión intracraneal en determinados síndromes craneofaciales y la aparición de pseudomeningoceles y fracturas evolutivas... Todos ellos son factores que se han relacionado con la aparición de defectos craneales postquirúrgicos ${ }^{1}$.

Se han empleado una gran variedad de materiales para reconstruir éstos defectos, tanto autólogos (injertos de calota tricortical desdoblada, costilla, peroné, viruta ósea con factores de crecimiento óseo...) como heterólogos (cementos de hidoxiapatita, resinas acrílicas, polietilenos o compuestos cerámicos) $)^{1,2}$. A las indudables ventajas de los injertos autólogos se asocia la dificultad para obtener suficiente cantidad de hueso dado el pequeño tamaño de las zonas donantes y los riesgos de interactuar con los cartílagos de crecimiento que afectarían el desarrollo óseo. Los injertos de materiales heterólogos tienen importantes limitaciones en la edad pediátrica. Existen contraindicaciones para el empleo de los mismos en edades tempranas $\mathrm{o}$ en defectos craneales completos, y siempre el riesgo de extrusión e infección por la ausencia o dificultad de osteointegración.

Solís y cols presentan su experiencia con la técnica de morcelización ósea con adhesivo de fibrina. Esta técnica popularizada en los últimos años por el grupo de Great Ormond Street (RD Hayward y cols) ${ }^{2}$ comenzó a utilizarse en la década de los 90 y existe una amplia experiencia en cuanto a su efectividad y seguridad ${ }^{3,4,5}$. Ha demostrado su utilidad a medio y largo plazo sobre todo en los casos de una primera intervención, donde la transposición de fragmentos tras las craneotomías y orbitotomías puede dejar defectos tras la remodelación tridimensional. Sin embargo, en nuestra experiencia, su utilidad es algo menor en los casos de reintervención y en pacientes de mayor edad, donde las cicatrices durales o la ausencia de periostio disminuyen la capacidad osteogénica y osteoinductora-integradora de ésta mezcla que tiende a reabsorberse prematuramente. En muchos de éstos casos debemos recurrir al injerto autólogo (fundamentalmente desdoblamiento de calota o injertos de costilla cuando aquél no sea posible) o en los casos de grandes defectos a plastias de material acrílico o cerámico como única opción. Como apuntan los autores en la discusión, sería interesante observar la evolución y osificación secundaria de la técnica en estudios de neuroimagen en éstos pacientes más comprometidos.
El mejor tratamiento de las craneolacunias persistentes es su prevención mediante una exquisita técnica quirúrgica, con la preservación tisular y especial atención al periostio y duramadre, la ausencia de complicaciones infecciosas y una adecuada planificación de la reconstrucción que minimice el tamaño de los defectos craneales tras la remodelación craneal.

Respecto a la afirmación de los autores de que los 9 meses es la edad idónea para la realización del tratamiento de la craneosinostosis, no podemos dejar de considerar el aserto como excesivamente estricto. Es posible que en el tratamiento de la plagiocefalia o de la braquicefalia por una sinostosis bicoronal no sindrómica éste criterio se aproxime a nuestra actitud. Sin embargo, cada paciente con una craneosinostosis debe ser considerado individualmente y en función de la técnica que pretendemos aplicar para su corrección. En ocasiones recomendaremos una cirugía precoz (suturectomías endoscópicamente asistidas en torno a los tres o cuatro meses de edad o escafocefalias en torno a los cinco meses, por ejemplo) mientras que en otros casos demoraremos el tratamiento en función de la aparición o no de síntomas de hipertensión intracraneal o variabilidad fenotípica de los pacientes (síndromes de Apert o Crouzon) $)^{1,6}$. Coincidimos con la opinión de los autores, que es mayoritaria en la bibliografía, de que el primer trimestre de vida debe evitarse debido a la cantidad de factores fisiológicos (anemia del recién nacido, vía aérea, persistencia de hemoglobina fetal con mayor afinidad por el $\mathrm{O}_{2}$ y por tanto peor liberación tisular, inmadurez e inestabilidad hemodinámica, menor volumen sanguíneo, pobre capacidad hemostática....) y anestésicos implicados. Una excepción a ésta regla son los síndromes craneofaciales graves con compromiso neurológico por hipertensión intracraneal, de vía aérea, o riego de lesión ocular por exposición de los globos.

El paciente de cirugía craneofacial es un individuo complejo, afectado por una enfermedad con múltiples implicaciones funcionales y estéticas, que requiere una valoración por diferentes especialistas y un tratamiento multidisciplinar que sólo puede y debe ser llevado a cabo en el seno de un grupo de trabajo que acumule la experiencia y sea capaz de ofertar todas las posibilidades terapéuticas existentes en cada momento.

\section{Bibliografía}

1. Esparza, J., Hinojosa, J.: Complications in the surgical treatment of craniosynostosis and craniofacial syndromes: apropos of 306 transcranial procedures. Child's Nerv Syst. 2008; 24: 1421-1430.

2. Raschid, A., Marucci, D.D., Dunaway, D.J., Hayward, R.D.: Bone 'salami': morcellised bone and fibrin glue for filling extensive cranial defects in craniofacial surgery. J Plast 
Reconstr Aesthet Surg. 2008; 61: 993-996.

3. Marchac, D., Renier, D.: Fibrin glue in craniofacial surgery. J Craniofac Surg. 1990; 1: 32.

4. Matsumoto, K., Kohmura, E., Kato, A., et al.: Restoration of small bone defects at craniotomy using autologous bone dust and fibrin glue. Surg Neurol 1998; 50: 344.

5. Thorn, J.J., Sorensen, H., Weis-Fough, U., et al.: Auto- logous fibrin glue with growth factors in reconstructive maxillofacial surgery. Int J Oral Maxillofac Surg. 2004; 33: 95.

6. Raised intracranial pressure in Apert syndrome. Marucci DD, Dunaway DJ, Jones BM, Hayward RD. Plast Reconstr Surg. 2008; 122: 1162-1168.

J. Hinojosa 